

\title{
Large-Eddy Simulation of Turbulent Flow Over a Rough Surface
}

\author{
Zhengtong Xie, Peter R Voke, Paul Hayden and Alan G Robins \\ Fluids Research Centre, School of Engineering, The University of Surrey, U.K.
}

August 6, 2003

\begin{abstract}
A family of wall models is proposed that exhibit more satisfactory performance than previous models for the large-eddy simulation of the turbulent boundary layer over a rough surface. The time and horizontally averaged statistics such as mean vertical profiles of wind velocity, Reynolds stress, turbulent intensities, turbulent kinetic energy and also spectra are compared with wind tunnel experimental data.

The purpose of the present study is to obtain simulated turbulent flows which are comparable with wind-tunnel measurements for use as the wind environment for the numerical prediction by LES of source dispersion in the neutral atmospheric boundary layer.
\end{abstract}

Keywords: large-eddy simulation, wall model, roughness, subgrid-scale model

\section{Introduction}

Large-eddy simulation (LES), which is well established in the study of small-scale meteorological and environmental flow phenomena as well as for engineering (Gao and Voke, 1995; Lesieur and Métais, 1996; Yang and Voke, 2001), is increasingly applied to atmospheric boundary layer (ABL) flows (Mason and Thomson, 1992; Moeng, 1984; Nieuwstadt et al., 1992; Thomas and Williams, 1999; Meeder and Nieuwstadt, 2000; Sullivan et al., 1994). In this paper, we focus attention on the surface layer or inner layer of a neutral ABL in which the Coriolis force effects are negligible and which is accordingly comparable with available measurements. We shall also investigate the turbulence almost at the core of the ABL for validation, where again we neglect the Coriolis force and consider that the boundary layer is evolving under free conditions.

One of the greatest challenges in applying large-eddy simulation to high Reynolds number flow over rigid walls is the wall model. Three main reasons have been cited by Piomelli (1999):

1. The growth of the small scales is inhibited by the presence of the wall.

2. The exchange mechanisms between the resolved and subgrid scale are altered.

3. The length scale of the energy-carrying large structures is Reynolds-number dependent near the wall. 
These physical effects of course interact strongly with the subgrid modelling in the LES context. Most ABL flows are over rough surfaces whose characteristics differ considerably depending on whether they are land, vegetation canopy, or lake or sea surfaces. The fully rough boundary makes the problem even more complicated, raising the additional questions:

- How may the roughness drag forces be determined?

- How much is the wake produced by the roughness?

- What similarity relations should we use for the effects of roughness?

- How may the roughness effects be modelled efficiently and simply?

Experimental observations (Krogstad and Antonia, 1994; Krogstad et al., 1992) show that roughness elements enhance the vertical intensity (normalised by $u_{*}$ ) significantly over the wall, while the principal Reynolds shear stress displays a roughness-dependent increase and the longitudinal turbulence intensity distribution is essentially the same as for the smooth wall. Unfortunately, standard large-eddy simulation models are not able to predict such enhancements reliably; in particular, they tend to underestimate the vertical fluctuation. Mason and Thomson (1992) argued that in the neutral case the departure from the logarithmic mean velocity profile may arise because the Smagorinsky SGS model has not taken account of the fluctuation of the SGS stress, as subgrid-scale stresses should have stochastic fluctuations and these fluctuations lead to a backscatter of energy from subgrid scale. Their model (Mason and Thomson, 1992) re-injects the turbulent kinetic energy using stochastic backscatter, in which random forcing leads to an unsteady velocity field with a partially stochastic component. Xin and Voke (1999) introduced a vertical forcing term in the Navier-Stokes equations in the vicinity of the rough wall, though some constant parameters had to be determined by tuning their code and the size of the forcing term was found to be grid-dependent.

Brown et al. (2001) used a plant canopy model combined with the stochastic backscatter model to generate more satisfactory turbulent flow away from the plant canopy, rather than investigating the flow detail within the plant canopy, as did Schumann, Shaw and other researchers (Kanda and Hino, 1994; Patton, 1997; Shaw and Schumann, 1992; Su et al., 1998). Their aim was to provide a boundary condition to the flow aloft. They found the plant canopy model was resolution independent and hence can be considered as a wall-model-like technique. However, the values of parameters such as the canopy drag coefficient $C_{D}$, the canopy density $a$ and the depth of the canopy layer must be related to specific experiments.

Thomas and Williams (1999) developed a synthetic rough wall boundary condition based on the well-known Schumann (1975) wall model. They consider the instantaneous stress as a linear combination of the mean and fluctuating components, where the fluctuating part responds less to the roughness and consequently contributes less to the local shear stress than in the standard Schumann (1975) wall model.

Moeng (1984) described a different wall model, originally proposed by Wyngaard in a personal communication. Compared with the standard Schumann (1975) wall model, 
their condition (specifying the SGS vertical fluxes at the wall surface) took account of more fluctuation. It was actually applied in convective ABL flows, though in this case the roughness has less effect than in the neutral or stable ABL. Mason and Gallen's (1986) rough wall model also manages to take account of more fluctuation. The similarity with the wall model proposed by Thomas and Williams (1999) is particularly noted.

We can classify the above contributions to modelling in the vicinity of a rough wall into two groups, those that:

A. Enhance the fluctuating component of the SGS vertical fluxes at the wall surface.

B. Inject backscatter energy into the resolved velocity field.

Group $\mathbf{A}$ are much simpler and easier to implement and do not increase the computational expense significantly. Group B are more complex, since some parameters need to be determined by numerical experiment or otherwise. They are more computationally expensive and may occasionally give rise to numerical instability. Based on the Thomas and Williams (1999) wall model, we develop a family of wall models in which the weight coefficient $\beta$ can be estimated theoretically rather than determined by numerical experiments.

Finally, we mention that boundary layer simulations may be carried out in several ways. In the most rigorous approach, a very large computational domain is used and upstream flow conditions are imposed, so that the boundary layer develops in space (as well as possibly in time) undergoing transition before becoming fully turbulent further downstream. The computational cost of this approach is generally high.

A simpler approach, adopted here, involves using a periodic condition in the streamwise direction and a mean streamwise pressure gradient - a driving force - which is added to the momentum equation. This permits a smaller computational domain and hence very cost-effective computations. Moreover, fast Fourier transformation (FFT) can be used in the computation, making the numerical scheme accurate and efficient.

\section{Governing Equations}

We consider incompressible air flow over a rough surface at very high Reynolds number. As it is quite impossible to do direct numerical simulations of such flows, and Reynoldsaveraged Navier-Stokes equation methods may eliminate most fluctuation information, we resort to large-eddy simulation. An LES resolves only the large-scale fluid motions and models the subgrid-scale (SGS) motions through filtering the Navier-Stokes equations.

We solve the filtered Navier-Stokes equations,

$$
\begin{aligned}
\frac{\partial u_{i}}{\partial x_{i}} & =0 \\
\frac{\partial u_{i}}{\partial t}+\frac{\partial u_{i} u_{j}}{\partial x_{j}} & =-\left(\frac{\partial p}{\partial x_{i}}+\delta_{i 1} \frac{\partial P}{\partial x_{1}}\right)+\frac{\partial}{\partial x_{j}}\left(\tau_{i j}+\nu \frac{\partial u_{i}}{\partial x_{j}}\right) .
\end{aligned}
$$


The dynamical quantities, $u_{i}, p$ are resolved-scale (filtered) velocity and pressure respectively and $\tau_{i j}$ is the subgrid-scale (SGS) Reynolds stress. The density is taken as unity. $u_{1}, u_{2}$ and $u_{3}$ are respectively the streamwise, lateral and vertical velocity components. $\delta_{i 1}$ is the Kronecher-delta and $\nu$ is the kinematic viscosity. $\partial P / \partial x_{1}$ is the driving force, a constant mean streamwise pressure gradient.

In the $x_{1}$ and $x_{2}$ directions the flow is periodic. At the top of the domain, stress free conditions are imposed on $u_{1}$ and $u_{2}$ :

$$
\frac{\partial u_{1}}{\partial x_{3}}=\frac{\partial u_{2}}{\partial x_{3}}=0 ; \quad u_{3}=0 .
$$

The top boundary condition here is simple but widely used (Shaw and Schumann, 1992), (Moeng, 1984). Shaw and Schumann (1975) pointed out the artificial boundary condition may be interpreted as a strong inversion, though it could also be viewed as a strict symmetry condition imposed at the centreline of a channel flow. In the region near the top of the domain, the numerically simulated flow is no doubt somewhat different from the flow in the simulated wind tunnel boundary layer or the flow in the outdoor atmospheric boundary layer. In the near wall region or far from the top of domain, the turbulence scales are governed by the rough wall and the differences caused by the treatment of the upper boundary may be assumed to be smoothed out or "forgotten". Nevertheless the mean velocity and second order moments must be carefully verified up to some height of the boundary layer.

At the bottom boundary the condition $u_{i}=0$ may be prescribed, but it is clear we cannot use this condition to calculate the surface stress owing to the high Reynolds number. Instead, the wall model relates the surface stress to the tangential velocity components $u_{1}$ and $u_{2}$ at the first inner grid point. Most commonly these are based on the log law:

$$
\bar{u}_{1}=\frac{u_{*}}{k} \ln \frac{x_{3}}{z_{0}}
$$

where $\bar{u}_{1}$ is the time-averaged streamwise velocity, $u_{*}$ is the friction velocity, $x_{3}$ is the distance from the wall, $k$ the von Karmann constant and $z_{0}$ is the roughness length. Details of the wall model for determination of the surface stress will be given in section 4 .

The mean pressure gradient $\partial P / \partial x_{1}$ in (1) must balance the mean surface stress:

$$
D \frac{\partial P}{\partial x_{1}}=\rho\left(u_{*}\right)^{2}
$$

where $D$ is the boundary layer thickness.

\section{Subgrid-Scale Model}

The Equations (1) cannot be solved unless the subgrid stress tensor $\tau_{i j}$ is expressed in terms of the known quantities, which is called subgrid-scale (SGS) modelling (Ferziger, 1993; Sullivan et al., 1994). The most commonly used subgrid-scale models are based on 
the Boussinesq hypothesis, which originally related the Reynolds stress occurring in the time-averaged Navier-Stokes equations for turbulent flows to the mean strain-rate and an eddy viscosity. The subgrid stress tensor $\tau_{i j}$ is assumed to be the product of fluid strain obtained from the filtered velocity field and a subgrid eddy viscosity:

$$
\tau_{i j}-\delta_{i j} \tau_{k k} / 3=\nu_{s}\left(\frac{\partial u_{i}}{\partial x_{j}}+\frac{\partial u_{j}}{\partial x_{i}}\right)=2 \nu_{s} S_{i j}
$$

To quantify the subgrid viscosity $\nu_{s}$, we use the mixed-scale model (MSM) of Sagaut (1995); this model is found to be robust, efficient and less problematic in the near-wall regions than the Smagorinsky model. The MSM expresses the subgrid viscosity in terms of the local strain-rate scalar $S=\sqrt{2 S_{i j} S_{i j}}$, the subgrid kinetic energy $q$ and a filter width $\Delta$, as follows:

$$
\nu_{s}(\alpha)=c_{M} S^{\alpha} q^{\frac{1-\alpha}{2}} \Delta^{1+\alpha}
$$

Depending on the value of $\alpha$, different models are recovered. The Smagorinsky model corresponds to $\alpha=1$ and the subgrid energy model to $\alpha=0$; the standard MSM of Sagaut (1995) has $\alpha=1 / 2$ and the corresponding constant $c_{M}$ is equal to 0.064 . The filter width is evaluated in a standard manner as $\Delta=\left(\Delta x_{1} \Delta x_{2} \Delta x_{3}\right)^{1 / 3}$. The subgrid kinetic energy $q$ is estimated using a test filter as commonly employed in dynamic SGS procedures (Germano et al., 1991):

$$
q=\frac{1}{2}\left(u_{i}-\tilde{u}_{i}\right)^{2}
$$

where the test filter (represented by the tilde) is twice the scale of the grid; $\tilde{u}_{i}$ is the test-filtered resolved velocity. Numerically, a normalized weighting function is applied to $u_{i}$ and the velocities at its neighbouring grids. More weight is given to the closer grids to $u_{i}$. Since the test filter is chosen twice of the size of the grid, the neighbouging grids are chosen to be the grids next to the point of $u_{i}$ and $\tilde{u}_{i}$ is calculated by integrating all of the weighted resolved velocities. The availability of $q$ is also very useful for estimating the SGS contribution to the diagonal stresses.

\section{Wall Model}

To simplify the expressions in this section, $u, v$ and $w$ denote the streamwise, lateral and vertical velocities $u_{1}, u_{2}$ and $u_{3}$ respectively. Also, $u^{\prime}, v^{\prime}$ and $w^{\prime}$ denote the instantaneous fluctuations of these velocities around their respective mean values $\bar{u}, \bar{v}$ and $\bar{w}$ (time- or ensemble-averaged).

As discussed in the introduction, it is too expensive (and impossible for rough walls) to resolve the near wall region, since in this region the eddies are too fine. Modelling the flow in the vicinity of the wall (Ferziger, 1993) is therefore of great importance in the simulation of high Reynolds number flow. The wall model is in fact one of the keys to calculating the 
turbulent flow over a rough wall by LES. Simulations suggest that wall-region turbulence and turbulence far from the wall are relatively loosely coupled (Ferziger, 1993). Thomas and Williams (1999) developed a new wall model, similar to that proposed by Schumann (1975). These models assume that the instantaneous velocity at the grid point nearest a wall is exactly correlated with the shear stress the wall point directly below it (Ferziger, 1993). Thomas and Williams wall model is written as follows,

$$
\frac{\tau_{x z}}{u_{*}^{2}}=\frac{1}{U_{a}}[<u>+\beta(u-<u>)], \quad 0<\beta \leq 1.0,
$$

where $<>$ represents averaging over the horizontal plane in the flow field, assumed to be approximately equivalent to ensemble averaging. $U_{a}$ is the mean streamwise velocity $\bar{u}_{1}$ at the first grid location from the wall as derived from the $\log$ law (2), and $\tau_{x z}$ is the instantaneous principal shear stress $\left(\tau_{13}\right)$ at the same location. $\beta$ is a damping factor ranging from 0 to 1.

The idea behind the Thomas and Williams model is that the fluctuation $(u-<u>)$ does not contribute the same but in fact less $(\beta<1)$ to the local shear stress $\tau_{x z}$ than the mean part $\langle u\rangle$; physically, the fluctuation velocity reacts to the wall roughness less than the mean velocity. The local shear stress of a rough wall is mainly the impact force of the fluid's movement on the roughness elements, so the mean flow will always react to the existence of every roughness element. However since there are gaps between the roughness elements, fine eddies may last for a short time in these gaps and decay before they reach the roughness element. Fluctuations from these eddies will not interact with the roughness elements and hence contribute less to the local wall shear stress, which is why the contribution of fluctuations to local wall shear stress is damped in Equation (4).

Averaging Equation (4) over the horizontal plane, we find this wall model obeys the constraint:

$$
\frac{<\tau_{x z}>}{u_{*}^{2}}=\frac{<u>}{U_{a}} .
$$

To our knowledge, most wall models (Thomas and Williams, 1999; Moeng, 1984; Xin and Voke, 1999; Schumann, 1975) obey this constraint, though some wall models may not (Mason and Gallen, 1986).

In Thomas and Williams (1999) the damping factor $\beta$ is chosen to be 0.3 ; they found the results were satisfactory to suit their application. Nevertheless, the magnitude of $\beta$ remains an open problem. Here, we try to evaluate it approximately.

From Equations (4) and (5) we obtain

$$
\left(\tau_{x z}-<\tau_{x z}>\right)^{2}=\frac{u_{*}^{4}}{U_{a}^{2}} \beta^{2}(u-<u>)^{2} .
$$

Using the approximation that the spatial average $<>$ in the above equation is equal to the time average, represented by an overbar, the above relation can be rewritten

$$
\left(u^{\prime} w^{\prime}-\overline{u^{\prime} w^{\prime}}\right)^{2}=\frac{u_{*}^{4}}{U_{a}^{2}} \beta^{2} u^{\prime 2} .
$$


Averaging over time, we obtain

$$
\overline{\left(u^{\prime} w^{\prime}-\overline{u^{\prime} w^{\prime}}\right)^{2}}=\frac{u_{*}^{4}}{U_{a}^{2}} \beta^{2} \overline{u^{\prime 2}} .
$$

An empirical result due to Uberoi (1953),

$$
\overline{u^{\prime} w^{\prime} u^{\prime} w^{\prime}} \approx 2 \overline{u^{\prime} w^{\prime}} \overline{u^{\prime} w^{\prime}}+\overline{u^{\prime 2}} \overline{w^{\prime 2}},
$$

may be used to show that the left hand side of Equation (7) is approximately

$$
\overline{u^{\prime} w^{\prime} u^{\prime} w^{\prime}}-\overline{u^{\prime} w^{\prime}} \overline{u^{\prime} w^{\prime}} \approx \overline{u^{\prime} w^{\prime}} \overline{u^{\prime} w^{\prime}}+\overline{u^{\prime 2}} \overline{w^{\prime 2}} .
$$

Then Equation (7) can be rewritten as:

$$
\overline{u^{\prime} w^{\prime}} \overline{u^{\prime} w^{\prime}}+\overline{u^{\prime 2}} \overline{w^{\prime 2}}=\frac{u_{*}^{4}}{U_{a}^{2}} \beta^{2} u^{\prime 2} .
$$

We can now determine the factor $\beta$ as follows:

$$
\beta^{2}=\frac{U_{a}^{2}}{u_{*}^{2}} \frac{\overline{u^{\prime 2}}}{u_{*}^{2}}\left[1+{\overline{u^{\prime} w^{\prime}}}^{2} /\left(\overline{u^{\prime 2}} \overline{w^{\prime 2}}\right)\right] .
$$

We see from this that $\beta$ is a function of $\overline{w^{\prime 2}} / u_{*}^{2}$ and $U_{a}^{2} / u_{*}^{2}$. Typically in a neutral ABL, ${\overline{u^{\prime} w^{\prime}}}^{2} /\left(\overline{u^{\prime 2}} \overline{w^{\prime 2}}\right)$ is approximately 0.1 , which can be considered a negligible factor. Since $0<\beta \leq 1.0, U_{a}$ must be limited. The limitation of $U_{a}$ is realized by carefully choosing the normalized vertical coordinate of the first grid point $z_{a}^{+}$; thus $z_{a}^{+}$must be located in the logarithmic layer, for instance between 20 and 200, which is the conclusion of many other researchers. We note the limitation of $U_{a}$ is fairly strict in Equation (4). If there are available experimental measurements of $\overline{w^{\prime 2}} / u_{*}^{2}$, we can use this information here directly; if not, we have to use some similarity relations (Stull, 1993) for neutral ABL flow.

Although the wall model described above has successfully improved predictions of the enhancement of vertical mixing in the near wall region, the turbulent intensities in the three directions are not equally satisfactory when compared with wind-tunnel experimental data, especially for the lateral fluctuations. Thomas and Williams (1999) only compared the intensity of the streamwise fluctuations with experimental measurements. Furthermore, it is of importance to consider the different effect on the roughness elements of smaller and larger eddies in the near wall region. Smaller eddies in the gaps between roughness elements will have less impact on them and hence contribute less to the local wall shear stress than larger eddies. In other words, the impact on roughness should not be linear in the amplitude of fluctuation as implied by the linear combination of the mean and fluctuating components in the Thomas and Williams model.

In order to obtain more reliable statistics in the study of scalar dispersion, comparable with experiment, it is desirable to generate an even more realistic wind environment by LES. This can be achieved by choosing the damping factor in Equation (4) as a function 
$f(u-\langle u>)$ rather than a constant. $f(u-\langle u\rangle)$ should be an increasing and positive function. A simple function such as

$$
\beta \frac{\left[(u-<u>)^{2}\right]^{n / 2}}{u_{*}^{n}} \quad n \geq 0,
$$

is one choice, but might not be the best one. Hence, a general family of wall models is proposed, written as follows:

$$
\frac{\tau_{x z}}{u_{*}^{2}}=\frac{1}{U_{a}}\left[<u>+\beta \frac{\left((u-<u>)^{2}\right)^{n / 2}}{u_{*}^{n}}(u-<u>)\right], \quad n \geq 0
$$

Introducing the approximation (9) we get:

$$
\overline{u^{\prime 2}} \overline{w^{\prime 2}}+\left(\overline{u^{\prime} w^{\prime}}\right)^{2}=<\beta^{2} \frac{\left(u^{\prime 2}\right)^{2 n}}{\left(u_{*}^{2}\right)^{2 n}} u^{\prime 2}>
$$

As $\frac{<u^{\prime 2}>^{2 n}}{\left(u_{*}^{2}\right)^{2 n}}$ and $u^{\prime 2}$ are both non-negative, we assume that

$$
<\frac{\left(u^{\prime 2}\right)^{2 n}}{\left(u_{*}^{2}\right)^{2 n}} u^{\prime 2}>\approx \frac{<u^{\prime 2}>^{2 n}}{\left(u_{*}^{2}\right)^{2 n}}<u^{\prime 2}>
$$

The factor $\beta$ is then obtained as

$$
\beta^{2}=\frac{U_{a}^{2}}{u_{*}^{4}} \frac{\left(u_{*}^{2}\right)^{2 n}}{{\overline{u^{\prime 2}}}^{2 n}} \overline{w^{\prime 2}}\left[1+\overline{u^{\prime} w^{\prime}} /\left(\overline{u^{\prime 2}} \overline{w^{\prime 2}}\right)\right] .
$$

Setting $n=0$ recovers the model (11) of Thomas and Williams (1999). Models in the family with $n<2$ have been tried and are found to improve the distribution of energy among the components compared with $n=0$, though not enough to be fully satisfactory. The model with $n=2$ appears to be close to optimal, while models with $n>2$ give rise to excessively sharp peaks of the intensities near the wall. Setting $n$ to 2 , we obtain a value of $\beta$ as follows:

$$
\beta^{2}=\frac{U_{a}^{2} u_{*}^{4} \overline{w^{\prime 2}}}{{\overline{u^{\prime 2}}}^{4}}\left[1+{\overline{u^{\prime} w^{\prime}}}^{2} /\left(\overline{u^{\prime 2}} \overline{w^{\prime 2}}\right)\right]
$$

Since $\beta$ should be restricted not to exceed 1.0 in Equation (15), $U_{a}$ must be limited by choosing $z_{a}^{+}$between 20 and 200. Compared with Equation (11), there is an addition small factor $u_{*}^{2} \sqrt{u^{\prime 2}} \approx 1 / 6$ in Equation (15), which is a similarity relation in neutral conditions. Recalling that $\overline{u^{\prime} w^{\prime}} /\left(\overline{u^{\prime 2}} \overline{w^{\prime 2}}\right) \approx 0.1$, we see that there is more freedom in Equation (15) to chose $z_{a}^{+}$to satisfy $0<\beta \leq 1.0$ than in Equation (11). Note that in all these models, the roughness length $z_{0}$ influences the stress only via the log law (2) and the resulting value of $U_{a}$.

It seems reasonable to separate the fluctuating velocity into two parts, small components and large components, the former responding to the wall roughness much less than 
the latter and consequently contributing much less than the large components to the shear stress. Obviously, the expression (15) reduces the contribution of the small fluctuating components to the Reynolds shear stress to a greater extent than (11).

A more general parameterization of the wall model could be a power series expansion in the term $\beta(u-<u>)$. In order to obey the constraint Equation (5), it would be better to write this in the following form:

$$
\frac{\tau_{x z}}{u_{*}^{2}}=\frac{1}{U_{a}}\left[\left\langle u>+(u-<u>)\left(\beta_{0}+\beta_{1} \frac{|u-<u>|}{u_{*}}+\ldots+\beta_{n} \frac{|u-<u>|^{n}}{u_{*}^{n}}\right)\right], \quad n \geq 0\right.
$$

where the term with $\beta_{0}$ represents the contribution of the first order of longitudinal fluctuation, the term with $\beta_{1}$ represents the contribution of the second order, and so on. Setting all the other damping factors except $\beta_{n}$ to zero recovers Equation (12). Since it is very complicated to evaluate the damping factors $\beta_{0}, \beta_{1}, \beta_{2}$, etc. to take account all of these terms, we use only one term of the above power series expansion for simplicity, as if the other terms make very little contribution to the local wall shear stress.

Schumann's wall model and the Thomas and Williams wall model are both first order models. However, there are some wall models containing more than one term of Equation (16). Corresponding to Wyngaard and Moeng's (1984) wall model, the above expression can be rewritten as follows:

$$
\tau_{x z}=\frac{<\tau_{x z}>}{<u>}\left[\frac{<u>}{<S>} S+(u-<u>)\right],
$$

where $S=\left(u^{2}+v^{2}\right)^{1 / 2}$. If we consider only $u^{\prime}=u-<u>$ in Equation (17) as a variable, assumed much smaller than $\langle u\rangle$, we can rewrite Equation (17) by applying power series expansion to $\left(u^{2}+v^{2}\right)^{1 / 2}$ to first order,

$$
\tau_{x z}=\frac{<\tau_{x z}>}{\langle u>}\left[\frac{<u>}{<S>}\left(\left(<u>^{2}+<v>^{2}\right)^{1 / 2}+\frac{<u>u^{\prime}+u^{\prime 2}}{\left.\left(<u>^{2}+<v\right\rangle^{2}\right)^{1 / 2}}\right)+u^{\prime}\right] .
$$

Hence, Wyngaard and Moeng's wall model is a combination of first and second order; however it should be noted that the factor $\left(\beta_{0}\right)$ of the first order term is not a small parameter in the above equation.

Mason and Gallen's (1986) rough wall model can be rewritten as,

$$
\tau_{x z}=\frac{k^{2}}{l n^{2}\left(1+z_{a} / z_{0}\right)}\left(u^{2}+v^{2}\right)^{1 / 2} * u .
$$

Again, we rewrite Equation (18) by using power series expansion to first order,

$$
\tau_{x z}=\frac{k^{2}}{\ln ^{2}\left(1+z_{a} / z_{0}\right)}\left[\left(<u>^{2}+<v>^{2}\right)^{1 / 2}+\frac{<u>u^{\prime}+u^{\prime 2}}{\left(<u>^{2}+<v>^{2}\right)^{1 / 2}}\right]\left(<u>+u^{\prime}\right) .
$$

Hence, Mason and Gallen's rough wall model could be considered as a combination of first, second and third order; the factor $\left(\beta_{0}\right)$ of the first order is also not a small parameter. 
In the current paper, we mainly use a simplified wall model that only keeps the third order term with $\beta_{2}$ (written as $\beta$ in other places for simplicity), which is a third-order wall model. In the following sections, if we don't specify a wall model, we imply this new model. Compared with the Thomas and Williams wall model, this third order model can inject the energy of mixing farther from the wall.

\section{Numerical Scheme}

We solve the resolved-scale motions using the time-dependent incompressible NavierStokes equations and the mixed-scale SGS model. The equations of motion (1) for the resolved velocity are discretized in time using the Adams-Bashforth scheme, with time splitting:

$$
\begin{aligned}
\frac{\partial u_{i}^{k+1}}{\partial x_{i}} & =0 \\
\frac{u_{i}^{k+1}-u_{i}^{k}}{\Delta t} & =-\frac{1}{\rho}\left(\frac{\partial p^{k+1 / 2}}{\partial x_{i}}+\delta_{i 1} \frac{\partial P}{\partial x_{1}}\right)+\frac{3}{2} H^{k}-\frac{1}{2} H^{k-1}
\end{aligned}
$$

where

$$
H=-\frac{\partial u_{i} u_{j}}{\partial x_{j}}+\frac{\partial}{\partial x_{j}}\left[\left(\nu+\nu_{s}\right)\left(\frac{\partial u_{i}}{\partial x_{j}}+\frac{\partial u_{j}}{\partial x_{i}}\right)\right] .
$$

Note that $k$ indicates the time step and this scheme is second-order in time.

Equations (19) are discretized in space using a second-order central differencing finitevolume method. We use a staggered grid which is uniform in both $x_{1}$ and $x_{2}$ directions and slightly stretched in the $x_{3}$ direction. All quantities are advanced in time through time splitting with two steps, giving a second-order explicit scheme. In step 1,

$$
\frac{\hat{u}_{i}^{k+1}-u_{i}^{k}}{\Delta t}=-\frac{1}{\rho} \delta_{i 1} \frac{\partial P}{\partial x_{1}}+\frac{3}{2} H^{k}-\frac{1}{2} H^{k-1},
$$

and in step 2,

$$
\begin{aligned}
\frac{\partial u_{i}^{k+1}}{\partial x_{i}} & =0 \\
\frac{u_{i}^{k+1}-\hat{u}_{i}^{k+1}}{\Delta t} & =-\frac{1}{\rho} \frac{\partial p^{k+1 / 2}}{\partial x_{i}}
\end{aligned}
$$

where the pressure is obtained from

$$
\frac{1}{\rho} \frac{\partial^{2} p^{k+1 / 2}}{\partial x_{i}^{2}}=\frac{1}{\Delta t} \frac{\partial \hat{u}_{i}^{k+1}}{\partial x_{i}}=R^{k+1 / 2} .
$$

This approach is commonly termed a projection method, since the pressure is used to project the velocity at the new time into the zero-divergence subspace of velocity function 
space. As the flow is assumed to be periodic in the streamwise and lateral directions, a discrete fast Fourier transform can be used to solve the discrete Poisson equation for pressure very efficiently. The above equation is Fourier transformed in $x_{1}$ and $x_{2}$, separating it into decoupled discrete Helmholtz equations in the remaining variable $x_{3}$ (with modified wavenumbers). These equations are simple tridiagonal matrix problems, solved rapidly on vector or shared-memory machines. The inverse discrete Fourier transform then yields the physical pressure field needed for the zero-divergence projection of the velocity field via Equation (20).

\section{Wind Tunnel Experiments}

The experimental measurements were obtained in the Fluids Research Centre ' $A$ ' tunnel at the University of Surrey. The test section of this wind tunnel is $0.9 \mathrm{~m}$ (width) $\times 0.6 \mathrm{~m}$ (height) $\times 5 \mathrm{~m}$ (length), with free-stream turbulence intensity less than $0.1 \%$ and the maximum free stream velocity $30 \mathrm{~ms}^{-1}$. A fan is used to maintain free-stream air flow. An array of expanded metal roughness with a height of $5 \mathrm{~mm}$ is laid at the bottom of the wind tunnel to provide a rough surface, while a metal shark-tooth fence is mounted at the inlet. A 1.6 meter length downstream is chosen as the working section, where the roughness Reynolds number was approximately 20 . We consider the surface in this working section to be fully aerodynamically rough. The boundary layer in the wind tunnel is a simulated atmospheric boundary layer, generated by the shark-tooth fence at the inlet and the array of expanded metal roughness at the bottom. The ABL simulation was very carefully checked, with the boundary layer over the fetch of the experiment being fully investigated. At several typical streamwise and lateral positions, Reynolds shear stress, turbulent intensities and the depth of the boundary layer were checked and compared. Afterwards, an optimal condition was set. The depth(D) of the boundary layer is approximately $67 \%$ of the wind tunnel depth. There is a very slow growth of boundary layer depth with distance, less than $2.5 \%$ of the fetch of experiment. Figure 1 shows the comparison between our measured data and Fackrell and Robins's (1982) data. The latter was also obtained in a simulated wind tunnel boundary layer flow; the agreement is quite good except at the top of the boundary layer.

The coordinate system origin is located at the mid-point of the upstream lower edge of the working section, with $x$ pointing downstream and $z$ upwards. Vertical profiles of three instantaneous velocities were measured at $(x, y, z)=(148,0,6: 500),(992,0,6: 500)$, (992, 150, 6:500), (992, -150, 6:500) and (1400, 0, 6:500) mm using an x-wire system.

Three free-stream velocities of $4,6,10 \mathrm{~ms}^{-1}$ were used for validation. When measuring the mean velocity, Reynolds stress, turbulence intensities and other point statistics, the sampling frequency and sampling period were $200 \mathrm{~Hz}$ and greater than one minute respectively. The velocity autocorrelation and spectra were measured using a maximum sampling frequency of $2000 \mathrm{~Hz}$. Comparisons of variances and stresses measured at 200 and $2000 \mathrm{~Hz}$ suggest errors of $2 \%$ or less at the measuring point nearest the wall, though the measurements must of course be missing even higher frequency components. 


\section{Results and Discussion}

The aim of the numerical simulation is to generate a reliable wind environment for the study of dispersion from a point source. The numerical results are judged by comparison with the wind tunnel measurements. We choose the boundary layer depth $D$ and friction velocity $u_{*}$ as reference length and reference velocity. The computational domain size is $4 D \times 1.47 D \times D$. The roughness element height $z_{h}$ is $0.0125 D$ and the roughness length $z_{0}$ is $0.00114 D$. We discretised the computational domain on a fine mesh of $256 \times 128 \times 128$; a medium mesh of $128 \times 64 \times 64$ was also used for some comparisons. In the wall model, the power factor $n \geq 0$ was taken as 2. The damping factor $\beta$ is calculated from Equation (15) and is equal to 0.083 in the fine mesh cases and 0.25 in the medium mesh cases. For comparison, Thomas and Williams wall model was applied in one medium mesh case with the same damping factor $\beta=0.25$. However, if the damping factor is evluated for Equation (11), it is much larger by using the same grids.

The simulation flow field was initialized with a logarithmic profile of streamwise velocity, null vertical velocity and random lateral velocity whose magnitude was $3.5 \%$ of the maximum streamwise velocity. The LES was integrated over about $9 D / u_{*}$ before the statistics were calculated, with a maximum Courant number $u \Delta t / \Delta x$ of about 0.16. The mean velocities, turbulence intensities, shear stresses and turbulence kinetic energy were obtained by both spatially averaging in the horizontal plane and time averaging over a time period of $4 D / u_{*}$.

Figure 2 (left) shows the computed profile of mean streamwise velocity compared with the wind tunnel measurements in the whole boundary layer; Figure 2 (right) shows the surface layer, which is of principal concern for environmental predictions. The profile of black triangle symbols were obtained when measuring $u$ and $v$ by using the same $\mathrm{x}$-wire system which was intensively used to measure $u$ and $w$. While it is normalized by $u_{*}$ which was obtained when measuring $u$ and $w$, which might be one reason why there is some discrepancy between this profile and other measured data in the very near wall region in Figure 2 (right). To show the effects of the new wall model for roughness, we also plot a profile obtained using the Schumann (1976) wall model and a profile obtained using the Thomas and Williams wall model. Without the model for the effects of roughness on the boundary layer, there is poor agreement with experiment. There is very little difference between the results from the new wall model and those from the Thomas and Williams wall model in the surface layer. Near the top of the domain, the results from the Thomas and Williams wall model seem better than those from the new wall model, compared with measurements. However, this is unlikely to be genuine; because the damping factor $\beta$ is chosen to be 0.25 for comparison (much smaller than it should be in our simulation, see Equation 11) much fluctuation has been taken account of in the wall model, making the profile steeper. The effect can also be found in Figure 5, where there is a dramatic sharp peak near the wall in the results using the Thomas and Williams wall model.

We do notice a discrepancy between the data from the new wall model and the measurements in Figure 2 (left) near the top of the domain, which may be mainly due to the simple upper boundary condition employed. Note that the mean velocity is normalized by $u_{*}$, since only the near wall region (or at least far from the upper boundary) is intensively 
investigated. The advantage of this is that the mean velocity in the near wall region can be checked and compared very carefully; the disadvantage is that a very small change of $u_{*}$ will generate a large discrepancy of normalized mean velocity. In addition, the method for generating the simulated boundary layer generates a much deeper boundary layer in a limited downstream distance than a naturally developed boundary layer (Counihan, 1969). It is very difficult to numerically simulate the whole depth of this boundary layer accurately. For instance, at the top of boundary layer in the wind tunnel, the flow is in fact not free stream, but with fairly large turbulent intensities. We focus our interest on the near wall area or at least far from the upper boundary.

These profiles are also plotted in Figure 3 using a logarithmic scale, demonstrating fair agreement between experimental and numerical results. It is also evident that the agreement between the profile from Schumann's wall model and measurement is poor. The difference between the data using the new wall model and that using the Thomas and Williams wall model is quite small in the near wall region.

Figure 4 (left) shows the vertical profile of normalized Reynolds shear stress and the wind tunnel measured data in the whole ABL, while Figure 4 (right) shows the surface layer, where the SGS and resolved contributions to the total are also separated. Above the height $z / D=0.025$, the SGS contributions is less than $10 \%$ of the total, which shows LES has resolved most scales except in the very near wall region. We find the difference between the predictions on the fine mesh and those on the medium mesh is quite small. The data using the new wall model is an evident improvement compared with that by using the Thomas and Williams wall model. The agreement between the numerical results and the measurements is quite encouraging. Owing to the constant driving force $\partial P / \partial x$ (uniform in the vertical direction) in combination with the stress-free condition on the upper boundary, there is a nearly linear variation of stress with height and a peak of Reynolds shear stress to offset the driving force in the near wall region, whereas the wind tunnel boundary layer is generated by a method in which the pressure gradient is very small. The variation of shear stress with height in the experiment is not necessarily linear, particularly in the near wall region where the measured profile is a little flatter. However it is still nearly linear above $Z / D=0.2$. As is well known, in the surface layer of an outdoor ABL, the shear stress is nearly constant with height. In Figure 4, the experimental stress profile tends to zero near $z / D=0.8$, while in Figure $5,6,7$, the turbulent intensities at $z / D=0.8$ are not small. We consider the flow below $z / D=0.8$ of the simulated boundary layer be more reliable for comparison. The drop in measured values near the wall is likely to be affected by high-frequency loss, which cannot be corrected even by measurements at $2000 \mathrm{~Hz}$. The discrepancy mentioned can also be seen in Figure 4 (left) in the region far from the surface.

The vertical profile of normalized turbulence r.m.s. fluctuation in the streamwise direction, compared with experiment data, is presented in Figure 5. The numerical results are quite reasonable, though in the near-wall region the large-eddy simulations slightly overestimate $u_{r m s}$, even when we allow for high-frequency loss in the measurements. The peak value on the medium mesh at approximately $z / D=0.01$ is higher than the measurements and the fine mesh prediction. In Figure 6, 7, the fine mesh also improves the predictions compared with the medium mesh. The peak of the data using the Thomas and 
Williams wall model is sharper while there is an evident underestimate at approximately $z / D=0.1$. We notice that decreasing the damping factor $\beta$ increases the peak of the profile. We have also noticed that increasing the power factor $n \geq 0$ in Equation (14) increases the peak of $u_{r m s}$; we ascribe the overestimation of $u_{r m s}$ to the peak of the Reynolds shear stress which we have discussed for Figure 4. Nevertheless, compared with the results obtained without modelling the effects of roughness, these predictions are more than acceptable. Note that there is an almost constant value over much of the surface layer in Figure 5 (right).

The numerical results compared with experimental data for the normalized turbulence r.m.s. fluctuation in the vertical direction are plotted in Figure 6. The current LES, which implements our new wall model, hardly underestimates $w_{r m s}$ compared with the measurements, and has greatly improved the modelling of the effects of roughness. We note that the result on the fine mesh is slightly better than that on the medium mesh, while the subgrid contribution, $q / 3$ from Equation (3), is important for obtaining this quality of agreement. Compared with the data using the Thomas and Williams wall model, an improvement is seen in the medium-mesh case. As far as $v_{r m s}$ is concerned, Figure 7, the numerical predictions are less good, giving smaller values for the whole surface layer. Again it is important to account for the subgrid contribution. The fact that the new wall model achieves less in the lateral direction than in the vertical direction may be due to the shape of the expanded metal roughness elements used in the wind tunnel; our wall model does not considered this anisotropy of the roughness. Compared with the medium mesh, the fine mesh shows little improvement for the lateral intensity. The implication is that our wall model is still not perfect, although the focus of interest is on $u_{r m s}$ and $w_{r m s}$. Compared with the Thomas and Williams wall model, the new wall model has demonstrated an important improvement in the surface layer.

We note that in Figures 4 (right), 5 (right), 7 (right) and 6 (right) there are plateaux in the surface layer, essentially broad peaks extending from $z / D=0.02$ up to 0.1 . These have been found by many researchers (Stull, 1993) and are associated with similarity relations. Table I is a summary of the similarity parameters; the data from Stull (1993) were compiled from other sources, most of which were field observations.

Table I. Comparison of similarity parameters in the surface layer

\begin{tabular}{|c|c|c|c|}
\hline & $u_{r m s} / u_{*}$ & $v_{r m s} / u_{*}$ & $w_{r m s} / u_{*}$ \\
\hline LES (fine mesh, total) & 2.7 & 1.38 & 1.14 \\
\hline LES (medium mesh, resolved) & 2.69 & 1.27 & 1.02 \\
\hline Experiment at FRC & 2.4 & 1.6 & 1.2 \\
\hline Robins' experiment (1982) & 2.3 & 1.6 & 1.2 \\
\hline Stull (1993) & $2.47,2.49,2.55$ & $1.70,1.73,2.07,2.47$ & $1.0,1.3,1.58$ \\
\hline
\end{tabular}


In Figure 8, the vertical profile of turbulent kinetic energy is compared with the measurements; the SGS part $q$ from Equation (3), the resolved part and the total of the calculated TKE are shown separately. We note that the SGS part is less than $10 \%$ of the total TKE except in the region $z / D \leq 0.05$ : most of the TKE has been resolved by the LES. The agreement between the numerical results and measurements is quite good. However, we note LES underestimates the TKE in the near region up to $z / D=0.1$. We ascribe this mainly to the underestimation of $v_{r m s}$ in Figure 7 ; this underestimation does not affect the prediction of turbulence behaviour in the vertical and streamwise directions.

The probability density functions for streamwise, lateral and vertical velocity at $z=$ $0.4425 D$ obtained by the LES are compared with Gaussian fitted curves in Figure 9. All the PDFs are comparable with a Gaussian distribution, though both the lateral velocity $v$ and the vertical velocity $w$ have a very slight drift.

The normalized turbulence spectra for streamwise, lateral and vertical velocity from the LES, calculated from the time autocorrelations by FFT, are presented in Figure 10 compared with our experimental data. A slope of $-5 / 3$ is quite distinct, indicating a Kolmogorov inertial range for both LES and experimental data. The mesh resolution limit of $f \times D / u_{*}$ for the medium mesh cases, estimated from the Taylor hypothesis, is approximately 280 , but a rapid drop of the spectrum is evident before $100 f \times D / u_{*}$. This is observed in other finite-volume LES results (Thomas and Williams, 1999) and is associated with the finite width of the top-hat filter in spectral space. On the fine mesh the Kolmogorov inertial range is extended by half a decade compared with the spectrum from the medium mesh.

\section{Concluding Remarks}

The purpose of the present numerical study is to obtain turbulent flows which are comparable with the wind tunnel measurements and can be used as a wind environment for studies of scalar dispersion in the model ABL. Our interest is focused on the micro-scales rather than meso-scales in the ABL. Such a study can only be satisfactory if we model the effects of roughness elements properly.

Based on the physical observation that roughness elements enforce vertical flow mixing, we have proposed a family of wall models to take into account the effects of roughness, in which the coefficient $\beta$ can be estimated using similarity relations or from wind tunnel measurements. It is clear that more information on the roughness should be taken into account to model the effects of roughness properly.

Large-eddy simulations of the neutral ABL have been realised using the proposed model and the numerical results have been compared with experimental measurements with some success. The LES underestimates the lateral turbulent intensity and we conclude that it may be necessary to consider the anisotropy of the physical roughness to model its effects accurately. The choice of the power factor $n$ in Equation (14) (i.e. which member of the proposed family of wall models is most suitable for some particular case) also remains an issue. 
From the detailed comparisons we conclude that the overall agreement between the numerical and experimental results is reasonably good and the model proposed for the effects of roughness elements is justified. The large-eddy simulations described have been used to study dispersion of a passive scalar in the neutral rough surface boundary layer, with extrapolation of the threshold levels of extreme values using Extreme Value Theory. The results will be reported in a subsequent paper.

\section{Acknowledgments}

This project is supported by NERC under grant GR3/12954. Parts of the computing were conducted at the Manchester Computer Centre. Many simulations have been performed on the Fluids Research Centre Origin-2000 provided under the JREI scheme. We are grateful to the referees for their helpful comments. We also would like to thank Dr S. Xin for his valuable discussions.

\section{References}

Brown A.R., Hobson J.M. and Wood N.: 2001, 'Large-eddy simulation of neutral turbulent flow over rough sinusoidal ridges', Boundary-Layer Meteorol., 98, 411-441.

Counihan, J.: 1969, 'An improved method of simulating an atmospheric boundary layer in a wind tunnel', Atmos. Env., 3, 197-214.

Fackrell,J.E. and Robins, A.G. : 1982, 'Concentration fluctuations and fluxes in plumes from point sources in a turbulent boundary layer', J. Fluid Mech., 117, 1-26.

Ferziger, J.H.: 1993, 'Subgrid scale modelling', in Large-Eddy Simulation of Complex Engineering and Geophysical flows (ed. Galperin, B. \& Orszag S.) Cambridge University Press, U.K., pp 37-54.

Gao S. and Voke P.R.: 1995, 'Large-eddy simulation of turbulent heat transport in enclosed impinging jets', Int. J. of Heat and Fluid Flow, 16, 349-356.

Germano M., Piomelli U., Moin P. and Cabot W.: 1991, 'A dynamic subgrid-scale eddy viscosity model', Phy. Fluids A, 3, 1760-1770.

Kanda M. and Hino M.: 1994, 'Organized structures in developing turbulent flow within and above a plant canopy, using a large-eddy simulation', Boundary-Layer Meteorol., 68, 237-257.

Krogstad P.A. and Antonia R.A.: 1994, 'Structure of turbulent boundary layers on smooth and rough walls', J. Fluid Mech., 277, 1-21.

Krogstad P.A., Antonia R.A. and Browne L.W.B.: 1992, 'Comparison between rough- and smooth-wall turbulent boundary layers', J. Fluid Mech., 245, 599-617

Lesieur M., and Métais O.: 1996, New trends in large-eddy simulations of turbulence. Annu. Rev. Fluid Mech., 28, 45-82.

Mason P.J. and Gallen N.S.: 1986, 'On the magnitude of the subgrid-scale eddy coefficient in large-eddy simulations of turbulent channel flow', J. Fluid Mech., 162, 439-462.

Mason P.J. and Derbyshire S.H.: 1990, 'Large-eddy simulation of the stably-stratified atmospheric boundary layer', Boundary-Layer Meteorol., 53, 117-162.

Mason P.J. and Thomson D.J.: 1992, 'Stochastic backscatter in large-eddy simulations of boundary layers', J. Fluid Mech., 242, 51-78.

Meeder J.P. and Nieuwstadt F.T.M.: 2000, 'Large-eddy simulation of the turbulent dispersion of a reactive plume from a point source into a neutral atmospheric boundary layer', Atmos. Env., 34, 3563-3573.

Mittal R. and Moin P.: 1997, 'Suitability of upwind biased finite differences schemes for LES of turbulent flows', AIAA J., 35, no 8, 1415-1417. 
Moeng C.-H.: 1984, 'A large-eddy simulation model for the study of planetary boundary layer turbulence', J. Atmos. Sci., 41, no.13, 2052-2062.

Nieuwstadt F.T.M., Mason P.J., Moeng C.-H. and Schumann U.: 1992, 'Large-eddy simulation of the convective boundary layer: a comparison of four computer codes', Turbulent Shear Flows 8, (ed. Durst et al.) Springer-Verlag, pp. 343-367

Patton E.G.: 1997, 'Large-eddy simulation of turbulent flow above and within a plant canopy', Doctoral Dissertation, Univ. of California, Davis CA, U.S.A.

Piomelli U.: 1999, 'Large-eddy simulation: achievements and challenges', Prog. Aero. Sci. 35, 335-362.

Sagaut P.: 1995, 'Simulations numériques d'écoulements décollés avec des modèles de sous-maille', PhD Thesis, University of Paris VI, France.

Schumann U.: 1975, 'Subgrid-scale model for finite difference simulations of turbulent flows in plane channels and annuli', J. Comput. Phys. 18, 376-404.

Shaw R. H. and Schumann, U.: 1992, 'Large eddy simulation of turbulent flow above and within a forest', Boundary-Layer Meteorol., 61, 47-64.

Stull R. B.: 1993, An Introduction to Boundary Layer Meteorology, Kluwer Academic, the Netherlands, 637 pp.

Su H.-B., Shaw R.H., Paw U.K.T., Moeng C.-H. and Sullivan P.P.: 1998, 'Turbulent statistics of neutrally stratified flow within and above a sparse forest from large-eddy simulation and field observation', Boundary-Layer Met., 88, 363-397.

Sullivan P.P., McWilliams J.C. and Moeng C.-H.: 1994, 'A subgrid model for large-eddy simulation of planetary boundary layer flows', Boundary-Layer Meteorol., 71, 247-276.

Thomas,T.G and Williams J.J.R.: 1999, 'Generation of a wind environment for large-eddy simulation of bluff body flows', J. Wind Eng. 63 Indust. Aerodyn., 82, 189-208.

Townsend A.A.: 1976, The Structure of Turbulent Shear Flow, Cambridge University Press, 429 pp.

Uberoi M.S.: 1953, 'Quadruple velocity correlations and pressure fluctuations in isotropic turbulence', J. Aeronaut. Sci., 20, 197-204.

Xin S. and Voke P.: 1999, Large-eddy simulations of rough surface boundary layer flows, report MEFD/99.98, Fluids Research Centre, Univ. of Surrey, Guildford, U.K.

Yang Z.Y. and Voke P.: 2001, 'Large-eddy simulation of boundary layer separation and transition at a change of surface curvature', J. Fluid Mech., 439, 305-333. 

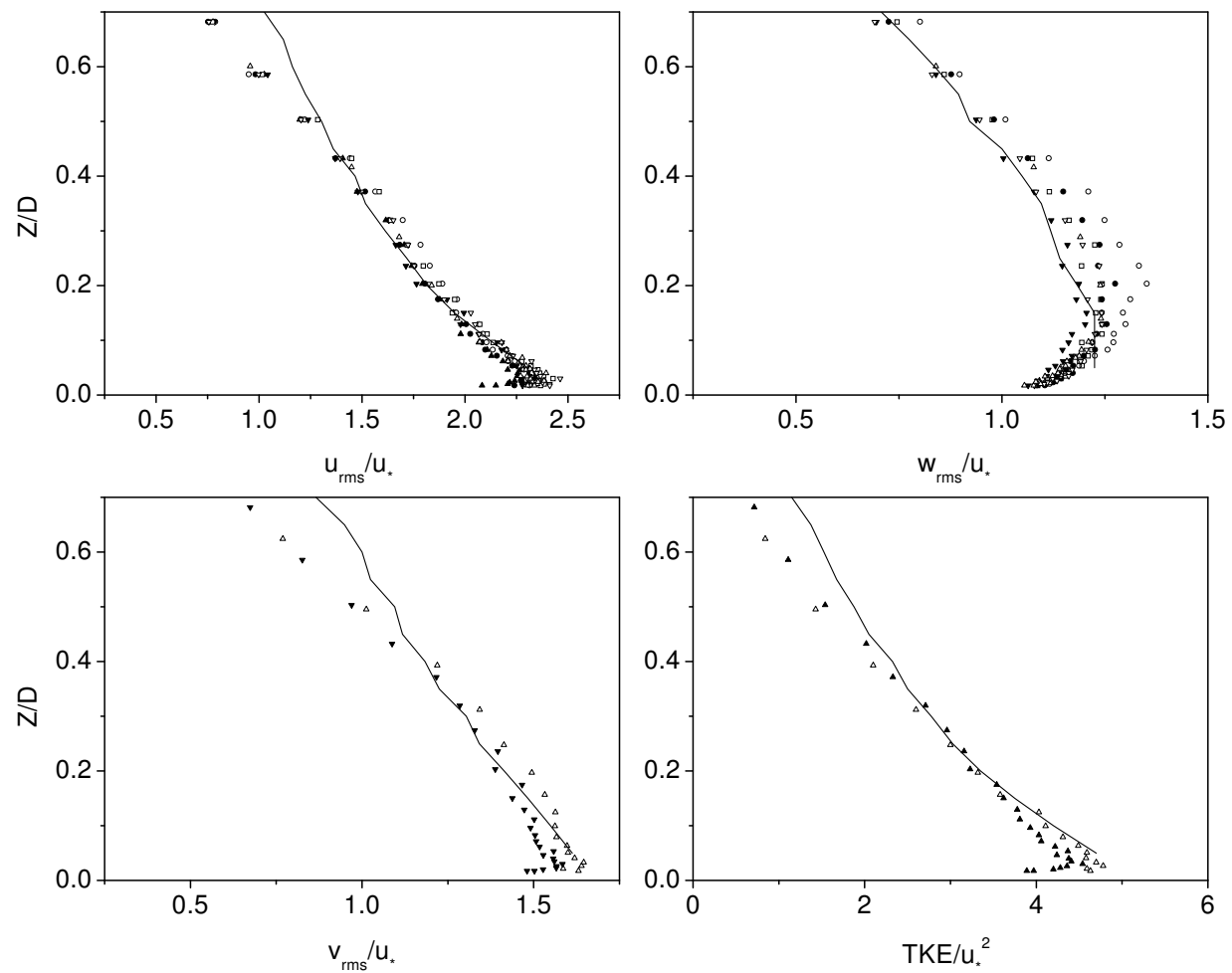

Figure 1. Turbulence fluctuations and TKE. $u_{r m s}=\sqrt{\left\langle u^{\prime 2}\right\rangle}, v_{r m s}=\sqrt{\left\langle v^{\prime 2}\right\rangle}, w_{r m s}=\sqrt{\left\langle w^{\prime 2}\right\rangle}$, $T K E=0.5\left(<u^{\prime 2}>+<v^{\prime 2}>+<w^{\prime 2}>\right)$. Symbols: measured data at several typical positions in wind tunnel at Surrey; Lines: Fackrell and Robins (1982) experimental data. 

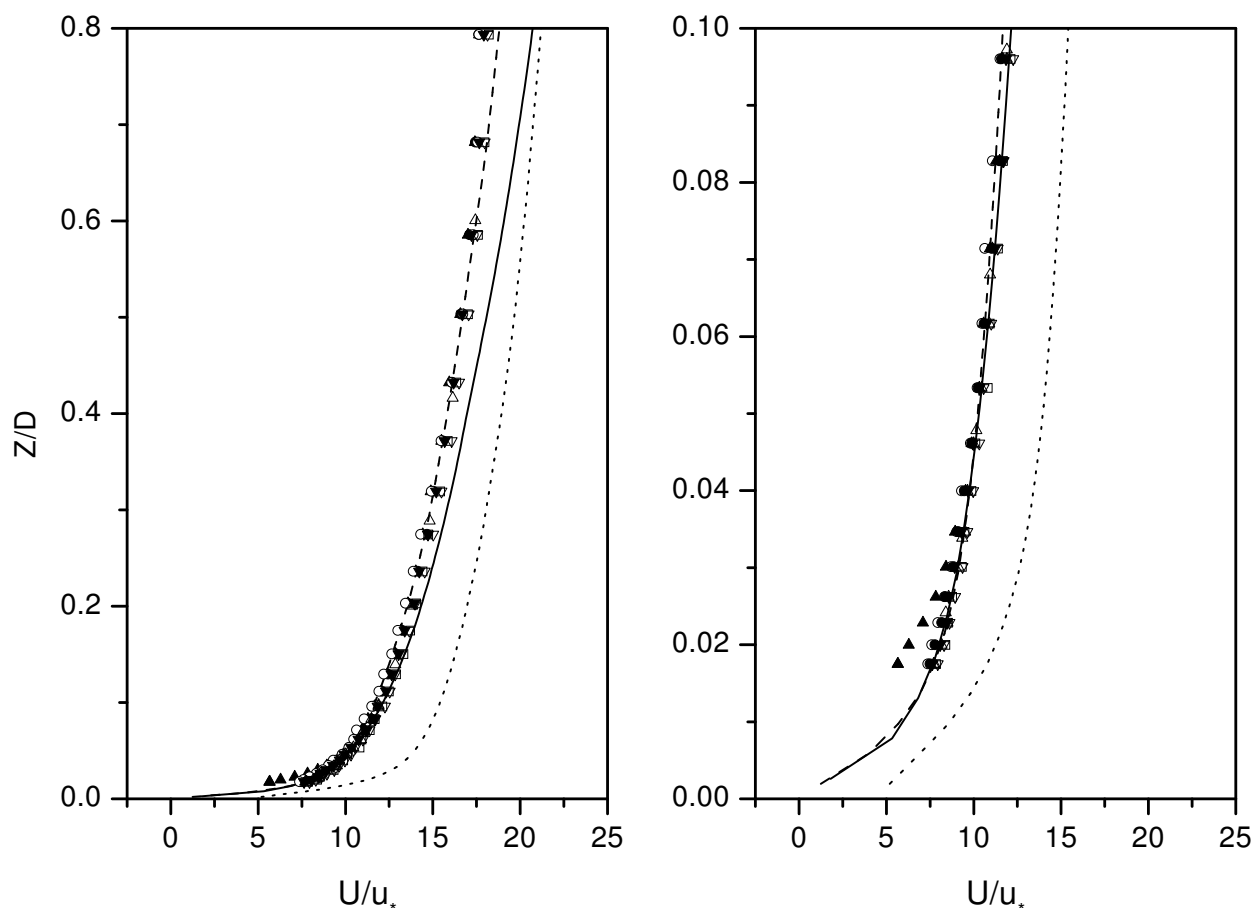

Figure 2. Streamwise mean velocity profiles. Lines, LES: solid, new wall model; dashed, Thomas and Williams wall model; dotted, Schumann's wall model; symbols, wind-tunnel measurements at several streamwise and lateral positions. (Right): whole boundary layer. (Left): surface layer. 

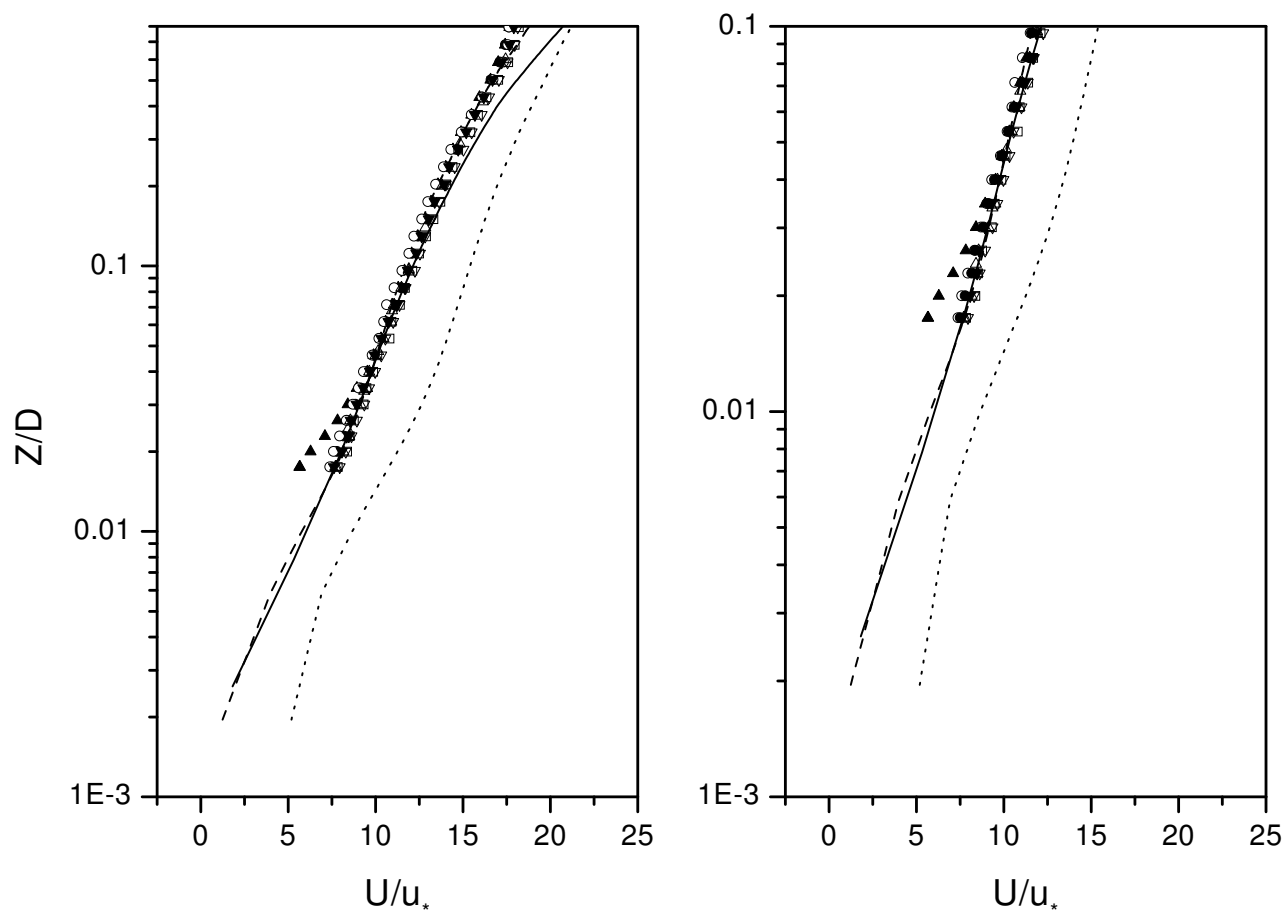

Figure 3. As Figure 1; logarithmic scale. 

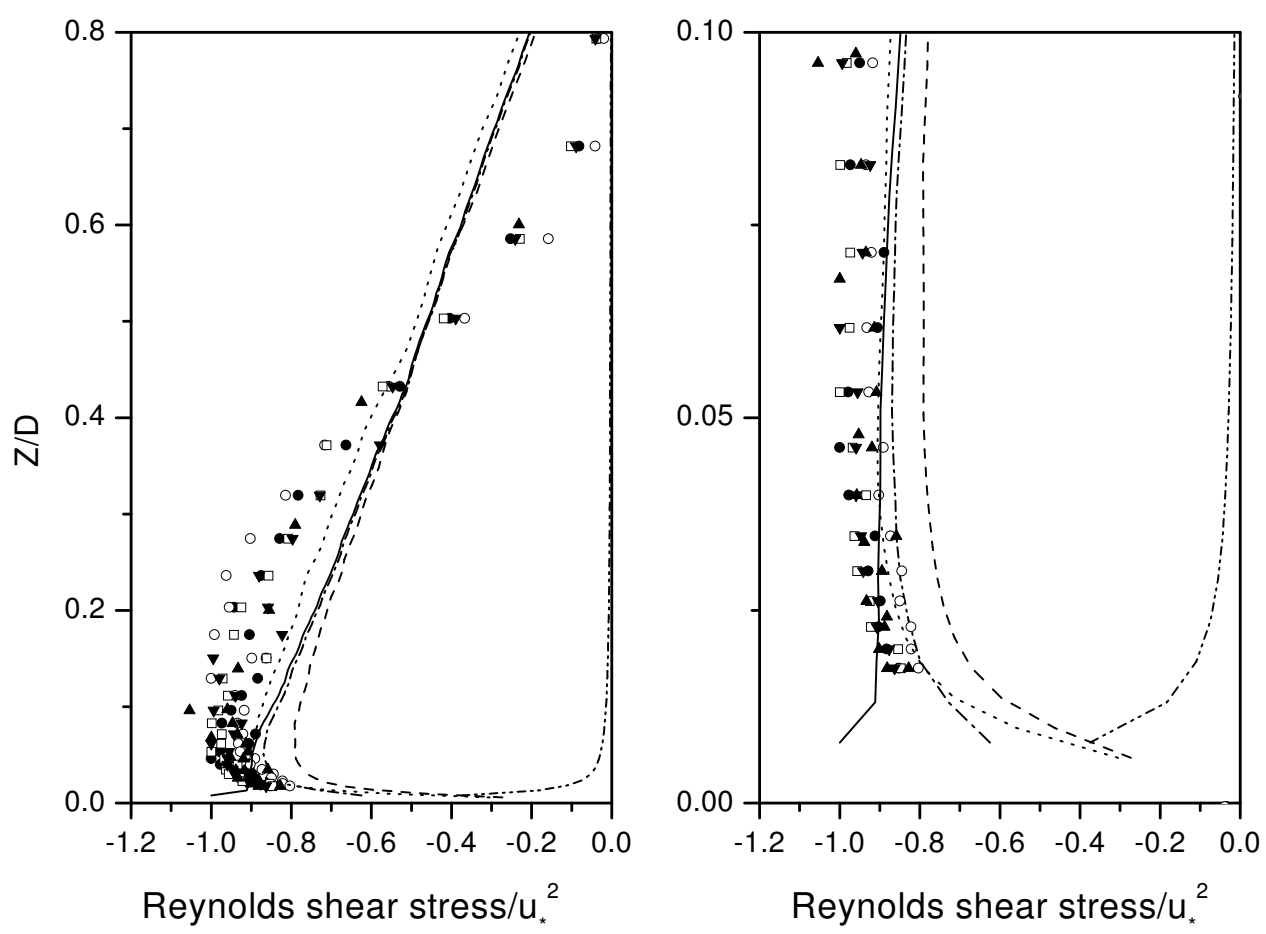

Figure 4. Reynolds shear stress profiles. Lines, LES: dash-dotted, fine mesh resolved; dash-dot-dotted, fine mesh sub-grid; solid, fine mesh total; dashed, Thomas and Williams wall model; dotted, medium mesh resolved; symbols, wind-tunnel measurements at several streamwise and lateral positions. (Right): whole boundary layer. (Left): surface layer. 

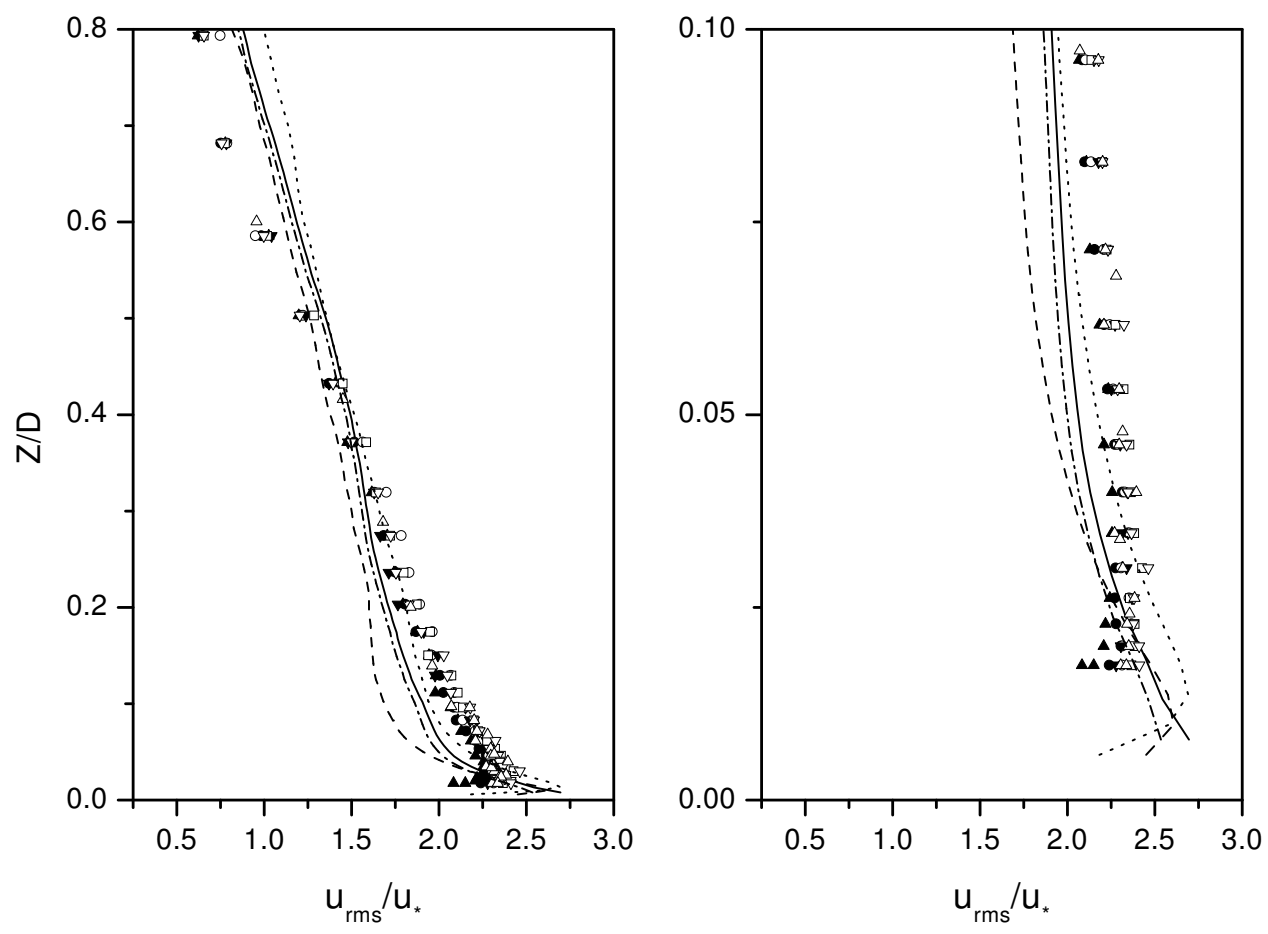

Figure 5. Turbulence fluctuation of streamwise velocity $u_{r m s}=\sqrt{\left\langle u^{\prime 2}\right\rangle}$. Lines, LES: dash-dotted, fine mesh resolved; solid, fine mesh total; dashed, Thomas and Williams wall model; dotted, medium mesh resolved; symbols, wind-tunnel measurements at several streamwise and lateral positions. (Right): whole boundary layer. (Left): surface layer. 

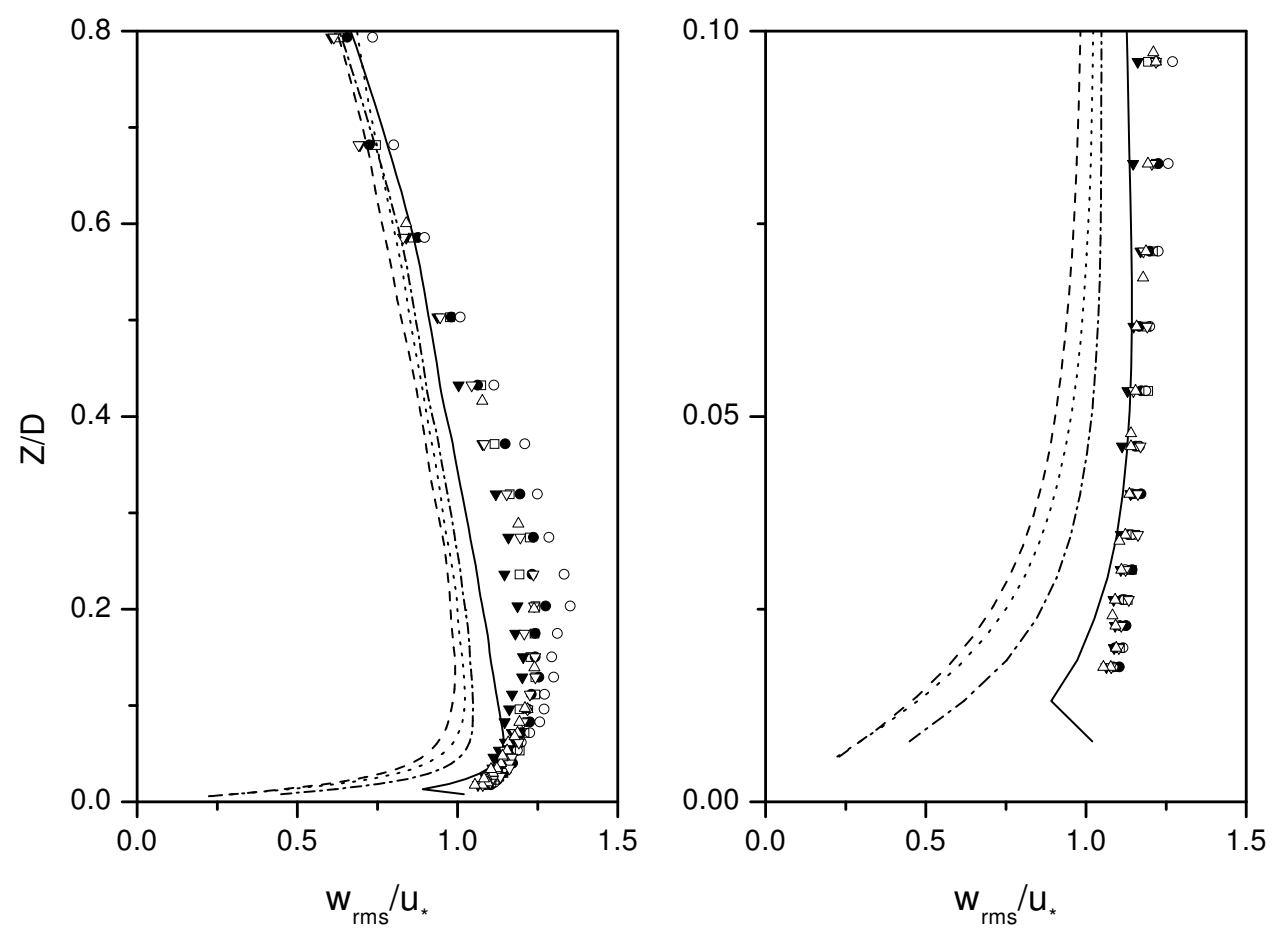

Figure 6. Turbulence fluctuation of vertical velocity $w_{r m s}=\sqrt{\left\langle w^{\prime 2}\right\rangle}$. As Figure (5). 

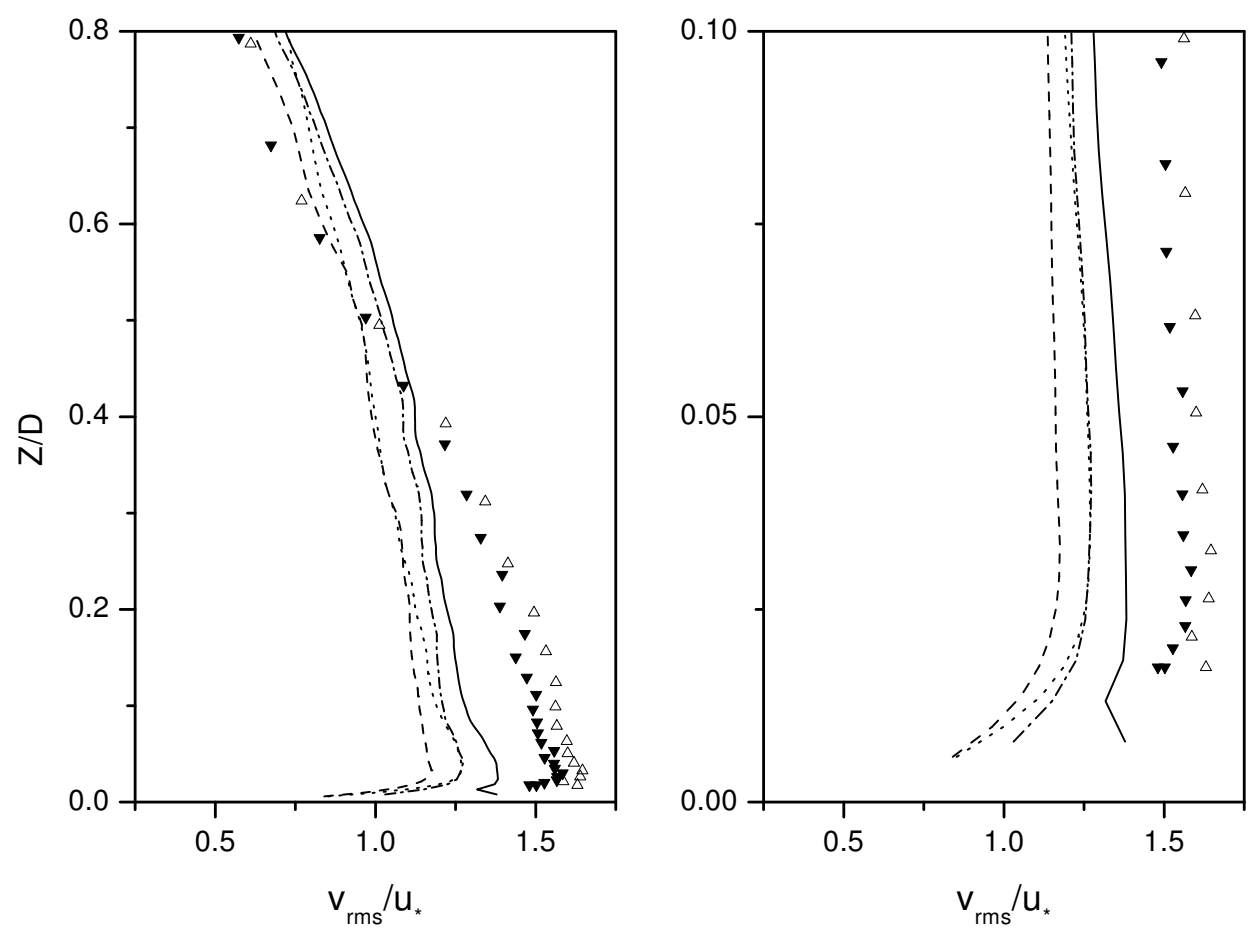

Figure 7. Turbulence fluctuation of lateral velocity $v_{r m s}=\sqrt{\left\langle v^{\prime 2}\right\rangle}$. As Figure (5). 

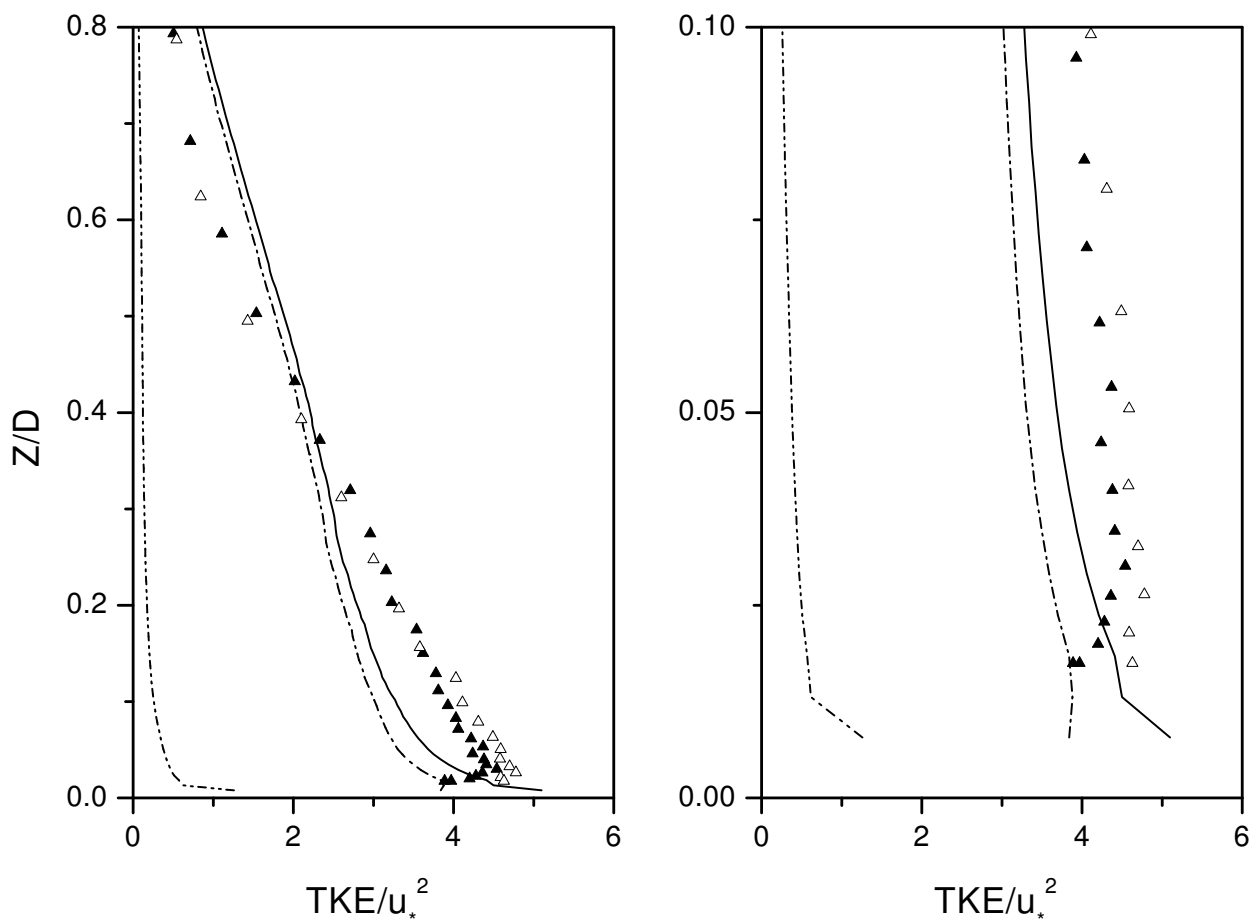

Figure 8. Turbulent kinetic energy profiles. Lines, LES: dash-dotted, fine mesh resolved; dash-dot-dotted, fine mesh sub-grid; solid, fine mesh total; symbols, wind-tunnel measurements at several streamwise and lateral positions. (Right): whole boundary layer. (Left): surface layer. 

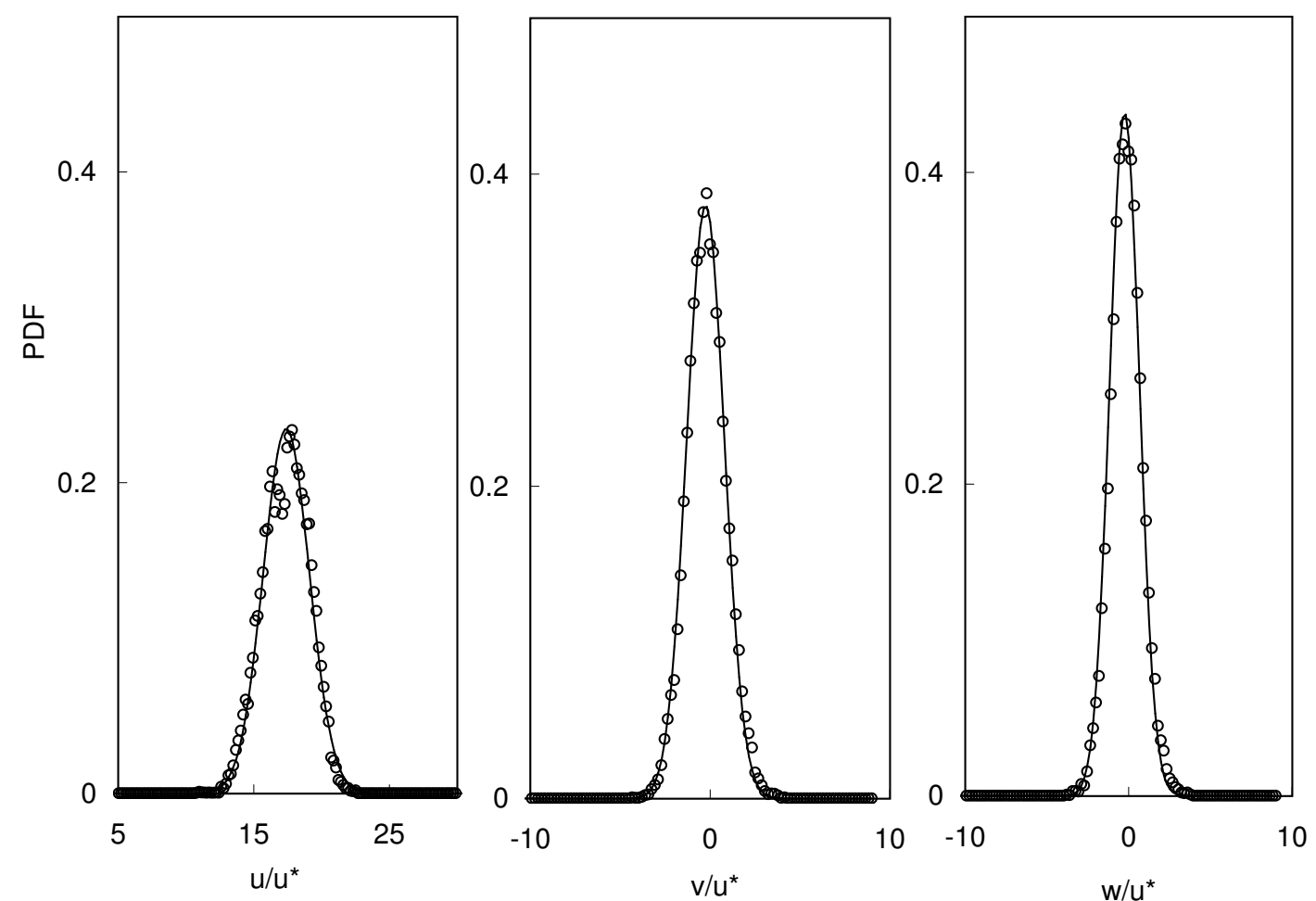

Figure 9. Probability density functions at height $z=0.4425 D$. Triangles, LES; solid lines, Gaussian-fit profiles. (Left): u. (Middle): v. (Right): w. 

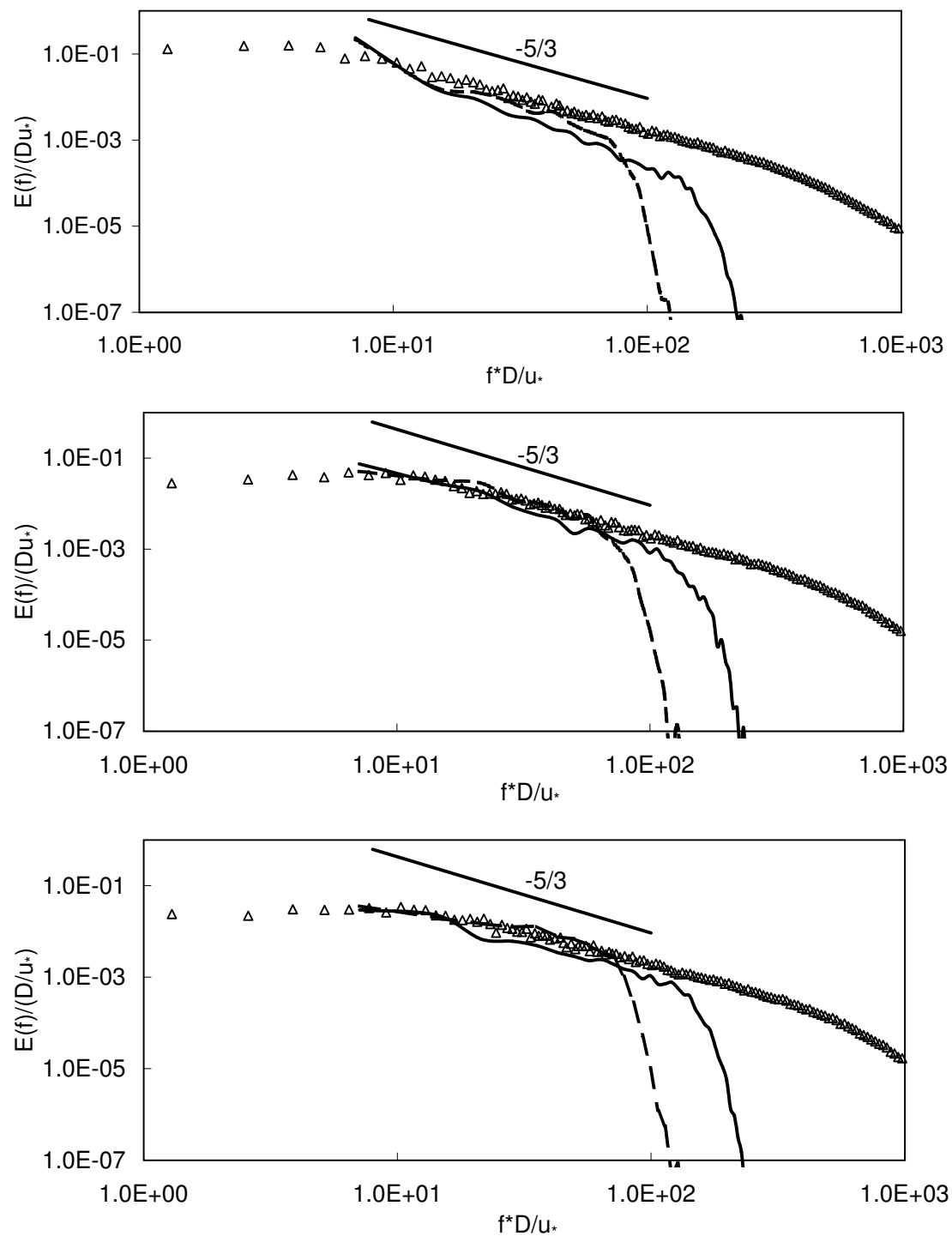

Figure 10. Normalised spectra. Lines, LES: solid, fine mesh; dashed, medium mesh; triangles, wind-tunnel measurements. (Top): u. (Middle): v. (Bottom): w. 
blm1fin.tex; 7/08/2003; 15:23; p.2 